\title{
Effect of rocuronium on the bispectral index under anesthesia and tracheal intubation
}

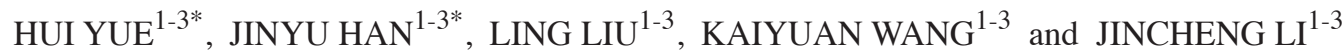 \\ ${ }^{1}$ Department of Anesthesiology, Tianjin Medical University Cancer Institute and Hospital; \\ ${ }^{2}$ National Clinical Research Center for Cancer; ${ }^{3}$ Key Laboratory of Cancer Prevention and Therapy, Tianjin 300060, P.R. China
}

Received May 7, 2015; Accepted June 2, 2016

DOI: 10.3892/etm.2016.3829

\begin{abstract}
The aim of the present study was to investigate the effect of various doses of rocuronium on bispectral index (BIS) responses to propofol induction and tracheal intubation, as well as the role of the non-depolarization muscle relaxant rocuronium on the depth of sedation. A total of 72 patients (American Society of Anesthesiologists physical status I-II) were anaesthetized with propofol using a target-controlled infusion, and randomly divided into two sedation level groups $(n=36)$. The patients were divided into 2 groups according to the BIS value: A normal sedation group (group 1), with a stable BIS value at 40-60, and a deep sedation group (group 2), with a BIS value $<20$ or with burst suppression. Each group was randomly divided into 4 subgroups A-D $(n=9)$ according to the various doses of rocuronium $(0.3,0.6,0.9$ and $1.2 \mathrm{mg} / \mathrm{kg})$. Tracheal intubation was performed after 2 min of rocuronium administration. BIS, electromyography (EMG), heart rate (HR) and mean arterial pressure (MAP) were recorded continuously and averaged over 1 min during baseline (T1), steady state (T2), 2 min after rocuronium infusion (T3), and 0,2 and 5 min after tracheal intubation. The results demonstrated that HR and MAP decreased significantly at T2 and T3 compared with T1. Following tracheal intubation (L0), HR and MAP significantly increased compared with T2 and T3, and returned to levels similar to those prior to intubation after 5 min. In group $1 \mathrm{C}$ and 1D, BIS was significantly decreased at T3 compared with T2; BIS was significantly increased at L0 compared with T3 in group 1A and 1B. EMG at earlier stages of anesthesia was significantly higher compared with other points, and was significantly increased at L0 compared with
\end{abstract}

Correspondence to: Professor Ling Liu, Department of Anesthesiology, Tianjin Medical University Cancer Institute and Hospital, Huan-Hu-Xi Road, Hexi, Tianjin 300060, P.R. China E-mail: liuling1244@163.com

*Contributed equally

Key words: rocuronium, bispectral index, anesthesia for induction, tracheal intubation
T3 in group 1A and 1B. These results demonstrated that BIS response may be associated with the dosage of rocuronium in the normal sedation group, although no association was observed with the deep sedation group. Tracheal intubation resulted in marked hemodynamic changes under both normal and deep sedation.

\section{Introduction}

As an important adjunct to anesthesia, muscle relaxants ensure that body movement does not occur in anesthetized patients and provide improved surgical conditions. Muscle relaxants are able to not only reduce the use of other anesthesia drugs, but also decrease the deleterious effects of deep anesthesia on the cardiovascular system (1). However, previous studies demonstrated that the probability of intraoperative awareness in patients administered muscle relaxants under general anesthesia was higher compared with patients who were not administered muscle relaxants $(2,3)$. In addition, patients treated with muscle relaxants showed increased postoperative injuries following intraoperative awareness during general anesthesia, as compared with patients who were not treated with muscle relaxants (4). Using electroencephalograph (EEG) algorithms to determine muscle activity, it has been observed that muscle relaxants exert some interference on the judgment and detection of anesthetic depth.

Bispectral index (BIS) is a signal processing technique combining EEG, electromyography (EMG) and a previously collected statistical database of patterns $(3,5)$. The BIS monitor reports a number from $0-100$, with 100 representing the awake state and 0 representing complete EEG inactivity (6). BIS is used to monitor the depth of anesthesia (4).

Two primary mechanisms have been proposed to underlie the effects of muscle relaxants on the depth of anesthesia: Firstly, the neuromuscular inhibition caused by muscle relaxants may directly affect the depth of anesthesia. Secondly, muscle relaxants inhibited EMG activities and influenced the monitoring of the depth of anesthesia, although muscle relaxants did not directly affect the depth of anesthesia. The first mechanism may be associated with a decrease in muscle-generated sensory inputs to the brain, and explains why rocuronium has been demonstrated to reduce halothane requirement and increase the depth of anesthesia (7). The second mechanism suggested that EMG activity just increased BIS values for general anesthetic 
patients, but their actual sedation depth was not changed $(8,9)$. In addition, the BIS significantly decreased and sedate status did not changed in patients in intensive care units, which may further confirm the validity of this mechanism (10).

Our previous studies demonstrated that propofol concentration and BIS correlated with the depth of sedation when propofol was used alone and in the absence of noxious stimulation, compound analgesics and muscle relaxants $(4,11)$. Muscle relaxants as anesthesia depth indicators may influence or interfere with the anesthetic dose and depth of sedation (5). Under a state of deep sedation, due to the inhibitions of EMG activity and afferent signals, muscle relaxants may lose their indicative role for anesthetic depth. Kawaguchi et al (12) reported that in the case of anesthesia using only propofol, a non-depolarizing muscle relaxant rocuronium would cause a dose-dependent inhibitory effect on the increase of entropy and BIS values following intubation.

The study hypothesized that under general anesthesia, propofol only induces both normal sedation and deep sedation. After various doses of muscle relaxants were administered and tracheal intubation was carried out, the changes in BIS and EMG were compared during the process of anesthesia induction, and prior to and following tracheal intubation. In addition, the mechanism underlying the effects of muscle relaxants on anesthesia depth was discussed.

\section{Materials and methods}

Patients. Ethical approval was obtained from the ethics committee of the Tianjin Medical University Cancer Institute and Hospital (Tianjin, China), and informed consents was obtained from the patients. A total of 72 patients (American Society of Anesthesiologists physical status I-II; age, 20-60 years) underwent anesthesia via endotracheal intubation for elective surgery. The exclusion criteria were as follows: Pregnancy; neurological, psychiatric and endocrine system diseases; cardiovascular and respiratory diseases; history of drug or alcohol dependency; neuromuscular blocking drug allergies; liver and kidney dysfunction; patients who were difficult to intubate and ventilate; and a body weight $>120$ or $<80 \%$ of the ideal body weight.

Data acquisition. None of the patients were medicated under any drugs. Following opening of venous access, administration of intravenous drip Lactated Ringer's solution (Otsuka Pharmaceutical Co., Ltd., Tianjin, China) and connection to Datex-Ohmeda S/5 monitoring equipment, electrocardiogram, non-invasive blood pressure and pulse oximetry finger were routinely monitored $(13,14)$. Subsequently, the foreheads of the patients were treated with alcohol, and a BIS VISTA $^{\mathrm{TM}}$ monitoring system-specific electrode was placed. Skin electrical impedance was maintained at $<5 \mathrm{ka}$. A BIS VISTA $^{\mathrm{TM}}$ monitor (Aspect Medical Systems, Inc., Natick, MA, USA) was connected to monitor the BIS. BIS and raw EEG data were collected in real-time using a RS232 serial port, with a sampling frequency of 128 samples/sec. The acquired data were used for subsequent analysis.

Anesthesia. Propofol (AstraZeneca, Basiglio, Italy) alone was administered by target-controlled infusion for anesthesia induction in all patients. The initial target concentration was $3.0 \mu \mathrm{g} / \mathrm{ml}$, until the patients lost consciousness and underwent mask ventilation. Target concentration increased to $0.5 \mu \mathrm{g} / \mathrm{ml} / 4 \mathrm{~min}$ to adjust the BIS value. The patients were randomly divided into 2 groups with 36 patients in each group. The BIS value was stable at 40-60 in group 1, which was termed the normal sedation group; the BIS value was $<20$ or with burst suppression in group 2, which was termed the deep sedation group. After maintaining the target BIS for $4 \mathrm{~min}$ in the 2 groups, the target plasma concentration and effect-site concentration reached a steady state, following which the target concentration of propofol did not change.

When they reached a steady state, the patients in the 2 groups were randomly divided into $\mathrm{A}, \mathrm{B}, \mathrm{C}$ and $\mathrm{D}$ subgroups $(n=9)$ according to the various doses $(0.3,0.6$, 0.9 and $1.2 \mathrm{mg} / \mathrm{kg}$ ) of rocuronium (Merck \& Co., Inc., Kenilworth, NJ, USA). Tracheal intubation was carried out 2 min after rocuronium administration. The BIS, EMG, heart rate (HR) and mean arterial pressure (MAP) were recorded prior to induction of anesthesia (T1), during the steady state (T2), following administration of rocuronium for $2 \mathrm{~min}$ (T3), and 0, 2, and $5 \mathrm{~min}$ following intubation (L0, L2 and L3, respectively). The target concentration of propofol was adjusted $5 \mathrm{~min}$ following the completion of intubation, and the BIS was maintained at 40-60 prior to the surgical procedure. Ephedrine (Northeast Pharmaceutical Group Co., Ltd., Shenyang, China) was administered to increase blood pressure when MAP was $<70 \mathrm{mmHg}$ and atropine (Tianjin Jinyao Amino Acid Co., Ltd., Tianjin, China) was administered to reduce the target concentration of intravenous anesthetics when HR $<50$ beats/min during induction. All the patients were kept at stable circulation with stable vital signs. The patients who showed marked body movement during intubation and those with an intubation time $>1$ min were not included in the statistical analysis.

Statistical analysis. Statistical analyses were performed using SPSS 17.0 (SPSS, Inc., Chicago, IL, USA). The data were expressed as means \pm standard deviation, and non-match two-tailed t-tests or two-way analysis of variance were used to analyze the difference. A Student-Newman-Keuls-q test was used to compare two groups; however, if the data was not normally distributed or exhibited heterogeneity of variance, a non-parametric test was used. The change in BIS and the doses of rocuronium were analyzed by the curve fitting. $\mathrm{P}<0.05$ was considered to indicate a statistically significant result.

\section{Results}

Patient information including age, weight and gender are presented in Table I, and were not significantly different between patients $(\mathrm{P}>0.05)$. Changes in $\mathrm{HR}$ and MAP prior to induction of anesthesia (T1), during steady state (T2), following administration of rocuronium for $2 \mathrm{~min}$ (T3), and 0,2 and 5 min following intubation (L0, L2 and L3, respectively) are presented in Tables II and III. The results demonstrated that compared with T1, HR and MAP at T2 and T3 significantly decreased $(\mathrm{P}<0.05)$. However, HR and MAP significantly increased at L0 compared with T2 and 
Table I. Patient information including age, weight and gender.

\begin{tabular}{llcc}
\hline Group & Age & Weight (kg) & Gender (man/woman) \\
\hline 1A & $48 \pm 4$ & $63.4 \pm 5.8$ & $5 / 4$ \\
1B & $41 \pm 8$ & $66.5 \pm 5.0$ & $6 / 3$ \\
1C & $44 \pm 8$ & $67.6 \pm 4.2$ & $6 / 3$ \\
1D & $43 \pm 6$ & $61.8 \pm 6.3$ & $4 / 5$ \\
2A & $46 \pm 4$ & $67.2 \pm 5.5$ & $5 / 4$ \\
2B & $45 \pm 8$ & $68.5 \pm 4.3$ & $6 / 3$ \\
2C & $46 \pm 5$ & $69.1 \pm 3.2$ & $5 / 4$ \\
2D & $50 \pm 3$ & $66.7 \pm 4.90$ & $6 / 3$ \\
\hline
\end{tabular}

Table II. Changes in HR prior to induction of anesthesia (T1), during steady state (T2), following administration of rocuronium for $2 \mathrm{~min}$ (T3), and 0,2 and 5 min after intubation (L0, L2 and L3, respectively).

\begin{tabular}{lcccccc}
\hline & & & & \multicolumn{3}{c}{ Following intubation } \\
\cline { 5 - 7 } $\begin{array}{l}\text { HR } \\
\text { (beats/min) }\end{array}$ & T1 & T2 & T3 & L0 & L2 & L5 \\
\hline 1A & $78 \pm 5$ & $70 \pm 10^{\mathrm{a}}$ & $72 \pm 3^{\mathrm{a}}$ & $92 \pm 7^{\mathrm{b}, \mathrm{c}}$ & $80 \pm 8$ & $72 \pm 8$ \\
1B & $82 \pm 3$ & $73 \pm 5^{\mathrm{a}}$ & $74 \pm 8^{\mathrm{a}}$ & $90 \pm 10^{\mathrm{b}, \mathrm{c}}$ & $78 \pm 6$ & $74 \pm 5$ \\
1C & $72 \pm 6$ & $66 \pm 4^{\mathrm{a}}$ & $70 \pm 3^{\mathrm{a}}$ & $85 \pm 6^{\mathrm{b}, \mathrm{c}}$ & $76 \pm 7$ & $68 \pm 7$ \\
1D & $76 \pm 7$ & $68 \pm 6^{\mathrm{a}}$ & $69 \pm 4^{\mathrm{a}}$ & $89 \pm 5^{\mathrm{b}, \mathrm{c}}$ & $70 \pm 3$ & $65 \pm 8$ \\
2A & $79 \pm 8$ & $62 \pm 5^{\mathrm{a}}$ & $64 \pm 8^{\mathrm{a}}$ & $74 \pm 3^{\mathrm{b}, \mathrm{c}}$ & $57 \pm 5$ & $52 \pm 4$ \\
2B & $80 \pm 5$ & $60 \pm 4^{\mathrm{a}}$ & $62 \pm 3^{\mathrm{a}}$ & $75 \pm 4^{\mathrm{b}, \mathrm{c}}$ & $64 \pm 7$ & $60 \pm 6$ \\
2C & $74 \pm 7$ & $55 \pm 7^{\mathrm{a}}$ & $59 \pm 6^{\mathrm{a}}$ & $69 \pm 7^{\mathrm{b}, \mathrm{c}}$ & $56 \pm 8$ & $58 \pm 3$ \\
2D & $70 \pm 6$ & $56 \pm 5^{\mathrm{a}}$ & $55 \pm 4^{\mathrm{a}}$ & $70 \pm 4^{\mathrm{b}, \mathrm{c}}$ & $54 \pm 5$ & $51 \pm 7$ \\
\end{tabular}

${ }^{\text {aP }}<0.05, \mathrm{HR}$ at $\mathrm{T} 2$ and $\mathrm{T} 3$ was significantly decreased compared with $\mathrm{T} 1 ;{ }^{\text {b,c }} \mathrm{P}<0.05, \mathrm{HR}$ at $\mathrm{L} 0$ was significantly increased compared with $\mathrm{T} 2$ and $\mathrm{T} 3$. HR, heart rate.

T3 $(\mathrm{P}<0.05)$. The change rate of hemodynamics in the deep sedation group was less marked compared with the normal sedation group. HR and MAP in each group returned to levels to those prior to intubation after $5 \mathrm{~min}$.

The changes in BIS in each group are presented in Fig. 1. The results demonstrated that BIS at T1 was significantly higher compared with the other time points $(\mathrm{P}<0.05)$. The BIS of groups $1 \mathrm{C}$ and $1 \mathrm{D}$ at T3 were significantly lower compared with T2 $(\mathrm{P}<0.05)$. The BIS of groups $1 \mathrm{~A}$ and $1 \mathrm{~B}$ at L0 were significantly higher compared with T3 $(\mathrm{P}<0.05)$. These results suggested that the BIS of groups $1 \mathrm{~A}$ and $1 \mathrm{~B}$ increased immediately following intubation.

The changes in EMG in each group are presented in Fig. 2. The results demonstrated that the EMG values at T1 were significantly higher compared with other time points $(\mathrm{P}<0.05)$. The EMG value of group $1 \mathrm{D}$ was significantly lower at T3, compared with T2 $(\mathrm{P}<0.05)$. The EMG values of groups $1 \mathrm{~A}$ and $1 \mathrm{~B}$ at L0 were significantly higher compared with T3 $(\mathrm{P}<0.05)$. These results suggested that the EMG of groups $1 \mathrm{~A}$ and $1 \mathrm{~B}$ increased immediately following intubation.
Table III. Changes in HR prior to induction of anesthesia (T1), during steady state (T2), following administration of rocuronium for $2 \mathrm{~min}$ (T3), and 0,2 , and $5 \mathrm{~min}$ following intubation (L0, L2 and L3, respectively).

\begin{tabular}{lcccccc}
\hline & & & & \multicolumn{3}{c}{ Following intubation } \\
\cline { 5 - 7 } $\begin{array}{l}\text { MAP } \\
\text { (mmHg) }\end{array}$ & T1 & T2 & T3 & L0 & L2 & L5 \\
\hline 1A & $109 \pm 5$ & $96 \pm 5^{\mathrm{a}}$ & $93 \pm 6^{\mathrm{a}}$ & $110 \pm 7^{\mathrm{b}, \mathrm{c}}$ & $100 \pm 6$ & $98 \pm 4$ \\
1B & $105 \pm 7$ & $89 \pm 2^{\mathrm{a}}$ & $90 \pm 7^{\mathrm{a}}$ & $108 \pm 8^{\mathrm{b}, \mathrm{c}}$ & $90 \pm 9$ & $86 \pm 3$ \\
1C & $103 \pm 4$ & $85 \pm 5^{\mathrm{a}}$ & $83 \pm 8^{\mathrm{a}}$ & $112 \pm 7^{\mathrm{b}, \mathrm{c}}$ & $93 \pm 4$ & $86 \pm 6$ \\
1D & $104 \pm 5$ & $86 \pm 4^{\mathrm{a}}$ & $87 \pm 6^{\mathrm{a}}$ & $115 \pm 5^{\mathrm{b}, \mathrm{c}}$ & $89 \pm 7$ & $84 \pm 4$ \\
2A & $101 \pm 8$ & $72 \pm 3^{\mathrm{a}}$ & $73 \pm 9^{\mathrm{a}}$ & $94 \pm 4^{\mathrm{b}, \mathrm{c}}$ & $84 \pm 5$ & $79 \pm 7$ \\
2B & $105 \pm 10$ & $77 \pm 6^{\mathrm{a}}$ & $75 \pm 4^{\mathrm{a}}$ & $96 \pm 8^{\mathrm{b}, \mathrm{c}}$ & $81 \pm 6$ & $76 \pm 5$ \\
2C & $103 \pm 4$ & $74 \pm 4^{\mathrm{a}}$ & $77 \pm 6^{\mathrm{a}}$ & $101 \pm 4^{\mathrm{b}, \mathrm{c}}$ & $85 \pm 4$ & $73 \pm 3$ \\
2D & $101 \pm 6$ & $73 \pm 5^{\mathrm{a}}$ & $71 \pm 3^{\mathrm{a}}$ & $92 \pm 6^{\mathrm{b}, \mathrm{c}}$ & $87 \pm 6$ & $72 \pm 5$ \\
\end{tabular}

${ }^{a}$ MAP at T2 and T3 significantly decreased compared with T1 $(\mathrm{P}<0.05)$; ${ }^{\mathrm{b}, \mathrm{c}} \mathrm{MAP}$ at L0 significantly increased compared with $\mathrm{T} 2$ and $\mathrm{T} 3(\mathrm{P}<0.05)$. MAP, mean arterial pressure.

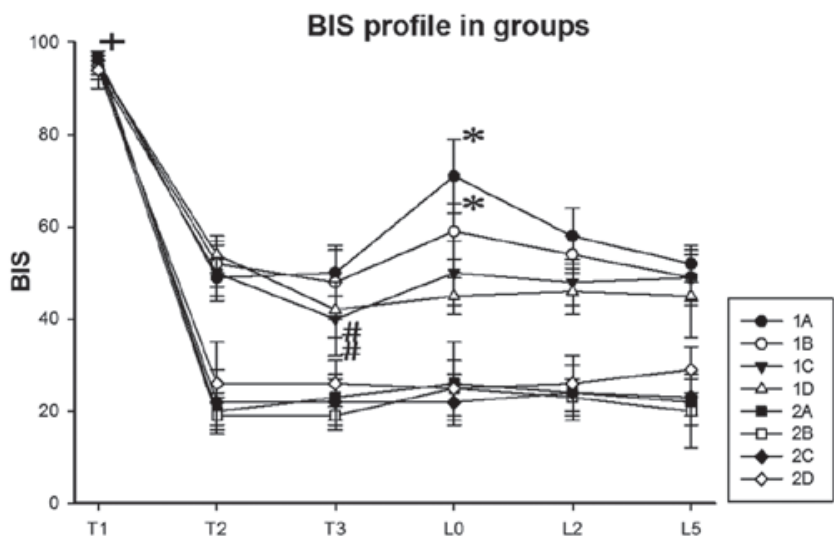

Figure 1. Changes in BIS in each group (means \pm standard deviation; $n=9$ ) prior to induction of anesthesia (T1), during steady state (T2), following administration of rocuronium for $2 \mathrm{~min}$ (T3), and 0,2 , and $5 \mathrm{~min}$ following intubation (LO, L2 and L3, respectively). ${ }^{+} \mathrm{P}<0.05$, BIS at T1 was significantly higher compared with the other time points; ${ }^{\#} \mathrm{P}<0.05$, BIS of groups $1 \mathrm{C}$ and $1 \mathrm{D}$ at $\mathrm{T} 3$ were significantly lower compared with $\mathrm{T} 2 ;{ }^{*} \mathrm{P}<0.05$, BIS of groups $1 \mathrm{~A}$ and $1 \mathrm{~B}$ at $\mathrm{L} 0$ were significantly higher compared with T3. BIS, bispectral index.

\section{Discussion}

With the development of various anesthesia drugs, novel muscle relaxants and anesthetic techniques have also been developed and applied; numerous clinical signs of general anesthesia are no longer regular and specific. Therefore, it is difficult to define the concept of general anesthesia and provide clear indications of a sedated state. Muscle relaxation lead to the decrease or disappearance of EMG near the electrodes, which in turn decreases the BIS. The indicative role of BIS on anesthesia depth is then affected. When monitoring indicators of depth of anesthesia, the numerical range of the depth of anesthesia (80-100, awake; 60-80, mild sedation; 40-60, anesthesia; $<40$, deep sedation) reflects the state of consciousness of a patient. A larger fluctuation range makes it more difficult to determine 
the state of consciousness of the patient using the index (15). Therefore, the present study aimed to study the impact and possible mechanism underlying the effects of muscle relaxants on depth of anesthesia.

Morimoto et al (16) investigated the association between BIS and EEG using nitrous oxide/isoflurane anesthesia. The authors developed a new index called burst-compensated spectral edge frequency 95\%, derived from the burst suppression ratio and spectral edge frequency, which is the product of the power spectrum analysis (16). The results revealed that BIS values may be calculated from Beta Ratios under shallow sedation (16). However, during deep anesthesia, synch fast slow (17) reflected power changes in EEG that were superior to changes in phase coupling in EEG. This suggests that synch fast slow predominantly reflected power changes in EEG, and BIS changes were largely due to power changes in EEG during deep anesthesia; and the impact of the EEG phase coupling is less marked than the EEG power.

Non-depolarizing muscle relaxants competed with acetylcholine for the nicotinic receptors, which are located at the motorial end-plate of striated muscles. Muscle relaxants cause muscle fibers to be in the polarization state and thus to have blocked ion channels. There were no end-plate potential activated excitement-contraction couplings, which suggests the blocked conduction between motor nerve terminals and striated muscles induced muscle relaxation. Previous studies have suggested that non-depolarizing muscle relaxants may have sedative effects $(18,19)$. Muscle stretch receptors activate the cerebral center, and non-depolarizing muscle relaxants inhibit the activity of stretch receptors, which results in sedation (20).

In the present study, patients were anesthetized with propofol using a target-controlled infusion, and these patients were randomly divided into 2 sedation groups. Each group was randomly divided into 4 subgroups A-D ( $n=9)$, according to the various doses of rocuronium used $(0.3,0.6,0.9$ and $1.2 \mathrm{mg} / \mathrm{kg}$ ). The results of the present study revealed that the BIS of groups $1 \mathrm{C}$ and $1 \mathrm{D}$ at $\mathrm{T} 3$ were significantly lower compared with T2 $(\mathrm{P}<0.05)$, and EMG changes were not statistically significant. This suggests that high doses of rocuronium effectively inhibited the activity of muscle stretch receptors, and afferent impulses from the muscle spindle to the wake center decreased. This resulted in the absence of painful stimulus and decreased BIS. These results were concordant with the hypothesis of muscle spindle afferents (21). Since there were no significant differences in EMG, the interference of frontal electromyography (fEMG) on BIS did not occur. Although EMG and BIS values in the other two groups $(0.3$ and 0.6 $\mathrm{mg} / \mathrm{kg}$ rocuronium) decreased, the differences were not statistically significant. Therefore, it may be hypothesized that rocuronium causes an afferent decrease in impulses from the muscle spindle to the wake center, which results in deeper sedation and decreased BIS.

EMG and BIS in the deep sedation group (group 2) at T2 and T3 exhibited no significant changes $(\mathrm{P}>0.05)$. This suggests that under deep sedation and in the absence of harmful stimuli, the inhibition of the central awakening by propofol was stronger than muscle spindle afferent impulses. The other possibility is that muscle relaxants eliminate EMG artifacts, which results in decreased BIS (22). fEMG may be inhibited by deep sedation which in turn counteracts rocuronium, thereby producing

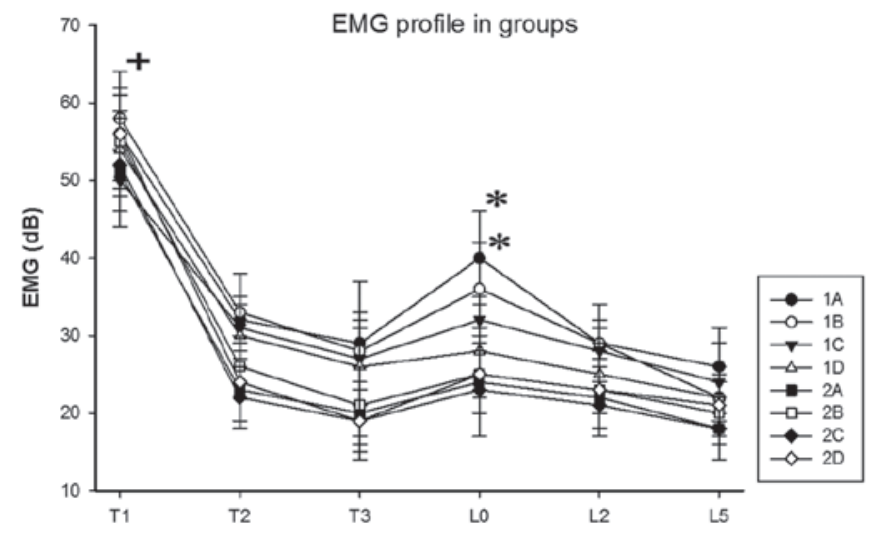

Figure 2. Changes in EMG in each group (means \pm standard deviation; $n=9$ ) prior to induction of anesthesia (T1), during steady state (T2), following administration of rocuronium for $2 \mathrm{~min}$ (T3), and 0,2 , and $5 \mathrm{~min}$ following intubation (L0, L2 and L3, respectively). ${ }^{+} \mathrm{P}<0.05$, EMG at $\mathrm{T} 1$ was significantly higher compared with other time points; ${ }^{*} \mathrm{P}<0.05$, EMG of groups $1 \mathrm{~A}$ and $1 \mathrm{~B}$ at L0 were significantly higher compared with T3. EMG, electromyography.

no statistically significant different in EMG and BIS. Muscle relaxants had no effect on BIS in the absence of EMG artifacts during deep sedation.

Endotracheal intubation is the most important harmful stimuli during anesthesia induction. This causes circulatory system fluctuations in the brain subcortical central (23). Cortical excitability awakes the patient (24), and may even lead to intraoperative awareness (25). Intubation during propofol anesthesia significantly increased BIS (4). However, other investigators observed that intubation did not have an impact on BIS, and may lead BIS to increase $(26,27)$. When sevoflurane is used alone, BIS was able to better monitor changes in the depth of anesthesia during endotracheal intubation; when combined with rocuronium, intubation conditions improved, the stress response caused by endotracheal intubation decreased, and the sensitivity of BIS decreased (28).

In the present study, BIS and EMG in groups $1 \mathrm{~A}$ and $1 \mathrm{~B}$ at L0 were significantly higher than at $\mathrm{T} 3(\mathrm{P}<0.05)$, and conversely EMG and BIS in groups $1 \mathrm{C}$ and $1 \mathrm{D}$ exhibited no statistically different changes $(\mathrm{P}>0.05)$. Various doses of rocuronium had different muscle paralysis effects. Rocuronium inhibited myoelectric activities following intubation, which suggested the increased BIS may be associated with rocuronium. Therefore, high doses of rocuronium did not significantly increase BIS; increased BIS caused by low doses of rocuronium may be associated with intubation stimulation and fEMG. Furthermore, when muscle activity was excited, the stretching or contraction of muscle fibers continued to increase muscle afferent activities, and cause afferent impulses caused excitation in the brain.

BIS and EMG in each group did not significantly change $(P>0.05)$ under deep sedation. This suggested that deep sedation inhibited the effects of intubation on BIS, results which are concordant with those of Morimoto et al (16). Under deep sedation, brain cell activity was inhibited, brain efficiency was decreased, and the inhibitory effect of propofol on brain awakening was significantly increased. Muscle spindle afferent impulses at this time may have been under the 
central brain arousal threshold, and the incoming excitatory stimulus was not sufficient to awaken the central nervous system (16).

BIS and hemodynamics are sensitive to stress response triggered by noxious stimuli such as endotracheal intubation and skin incisions (29). Guignard et al (24) also demonstrated that BIS was able to accurately reflect the degree of noxious stimuli during tracheal intubation. The results of the current study revealed that HR and MAP decreased in each group at T2 and T3, and significantly increased at L0 compared with T3 $(\mathrm{P}<0.05)$. However, changes in hemodynamics in the normal sedation group were significantly better compared with the deep sedation group. Rate-pressure in each group returned to the levels similar to those prior to intubation 5 min after intubation. This suggests that endotracheal intubation may result in significant hemodynamic changes, and HR and MAP increases both under general sedation and deep sedation. In addition, the various doses of rocuronium had no significant effect on hemodynamics.

Since this study was based on clinical research, following experimentation the patients remained sedated and underwent surgical procedures. Therefore, an awake rocuronium test group was not established. However, previous studies have reported that under BIS and EMG simultaneous monitoring, patients were able to communicate with the researchers when succinylcholine was given without any other anesthetics. These results revealed that BIS values decreased rapidly following administration of succinylcholine, which is similar to sedation under anesthesia. At the same time, fEMG activity disappeared. BIS recovery exhibited a correlation with EMG activity recovery (29). These results revealed that muscle relaxants alone are unable to sedate patients, however BIS decreased and induced a state of sedation.

\section{References}

1. Huang YF, Pryor ME, Mather LE and Veering BT: Cardiovascular and central nervous system effects of intravenous levobupivacaine and bupivacaine in sheep. Anesth Analg 86: 797-804, 1998.

2. Gazzanelli S, Vari A, Tarquini S, Fermariello A, Caputo M, Almansour M, Costi U, Basso L, Casullo L and Izzo L: Monitoring of consciousness with BIS during induction of anesthesia. G Chir 26: 163-169, 2005.

3. Gao JD, Zhao YJ, Xu CS, Zhao J, Huang YG, Wang TL, Pei L, Wang J, Yao LN, Ding Q, et al: Evaluation of entropy for monitoring the depth of anesthesia compared with bispectral index: A multicenter clinical trial. Chin Med J (Engl) 125: 1389-1392, 2012.

4. Hans P, Giwer J, Brichant JF, Dewandre PY and Bonhomme V: Effect of an intubation dose of rocuronium on spectral entropy and bispectral index responses to laryngoscopy during propofol anaesthesia. Br J Anaesth 97: 842-847, 2006.

5. Pomfrett CJ: Heart rate variability, BIS and 'depth of anaesthesia'. Br J Anaesth 82: 659-662, 1999.

6. Sie MY, Goh PK, Chan L and Ong SY: Bispectral index during modified rapid sequence induction using thiopentone or propofol and rocuronium. Anaesth Intensive Care 32: 28-30, 2004.

7. Forbes A, Cohen N and Eger EI II: Pancuronium reduces halothane requirement in man. Anesth Analg 58: 497-499, 1979.

8. Riess ML, Graefe UA, Goeters C, Van Aken H and Bone HG: Sedation assessment in critically ill patients with bispectral index. Eur J Anaesthesiol 19: 18-22, 2002.

9. Prell J, Rampp S, Ache J, Laule S, Rachinger J, Scheller C, Alfieri A and Strauss C: The potential of quantified lower cranial nerve EMG for monitoring of anesthetic depth. J Neurosurg Anesthesiol 24: 139-145, 2012.
10. Vivien B, Di Maria S, Ouattara A, Langeron O, Coriat $\mathrm{P}$ and Riou B: Overestimation of Bispectral Index in sedated intensive care unit patients revealed by administration of muscle relaxant. Anesthesiology 99: 9-17, 2003.

11. Park SJ, Cho YJ, Oh JH, Hwang JW, Do SH and Na HS: Pretreatment of magnesium sulphate improves intubating conditions of rapid sequence tracheal intubation using alfentanil, propofol, and rocuronium-a randomized trial. Korean J Anesthesiol 65: 221-227, 2013.

12. Kawaguchi M, Takamatsu I and Kazama T: Rocuronium dose-dependently suppresses the spectral entropy response to tracheal intubation during propofol anaesthesia. $\mathrm{Br} \mathrm{J}$ Anaesth 102: 667-672, 2009.

13. Pearsall FJ, Davidson JA and Asbury AJ: Attitudes to the Association of Anaesthetists recommendations for standards of monitoring during anaesthesia and recovery. Anaesthesia 50: 649-653, 1995.

14. Chun H, Kang J, Kim KJ, Park KS and Kim HC: IT-based diagnostic instrumentation systems for personalized healthcare services. Stud Health Technol Inform 117: 180-190, 2005.

15. Iannuzzi M, Iannuzzi E, Rossi F, Berrino L and Chiefari M: Relationship between bispectral index, electroencephalographic state entropy and effect-site EC50 for propofol at different clinical endpoints. Br J Anaesth 94: 492-495, 2005.

16. Morimoto Y, Hagihira S, Koizumi Y, Ishida K, Matsumoto M and Sakabe T: The relationship between bispectral index and electroencephalographic parameters during isoflurane anesthesia. Anesth Analg 98: 1336-1340, 2004.

17. Rampil IJ: A primer for EEG signal processing in anesthesia. Anesthesiology 89: 980-1002, 1998.

18. Hosseinzadeh $\mathrm{H}$ and Nassiri Asl M: Anticonvulsant, sedative and muscle relaxant effects of carbenoxolone in mice. BMC Pharmacol 3: 3, 2003.

19. Sanford TJ Jr, Weinger MB, Smith NT, Benthuysen JL, Head N, Silver $\mathrm{H}$ and Blasco TA: Pretreatment with sedative-hypnotics, but not with nondepolarizing muscle relaxants, attenuates alfentanil-induced muscle rigidity. J Clin Anesth 6: 473-480, 1994.

20. Dahaba AA, Mattweber M, Fuchs A, Zenz W, Rehak PH, List WF and Metzler H: The effect of different stages of neuromuscular block on the bispectral index and the bispectral index-XP under remifentanil/propofol anesthesia. Anesth Analg 99: 781-787, 2004.

21. Dahaba AA, Bornemann H, Hopfgartner E, Ohran M, Kocher K, Liebmann M, Wilfinger $\mathrm{G}$ and Metzler $\mathrm{H}$ : Effect of sugammadex or neostigmine neuromuscular block reversal on bispectral index monitoring of propofol/remifentanil anaesthesia. Br J Anaesth 108: 602-606, 2012.

22. Aho AJ, Kamata K, Yli-Hankala A, Lyytikäinen LP, Kulkas A and Jäntti V: Elevated BIS and Entropy values after sugammadex or neostigmine: An electroencephalographic or electromyographic phenomenon? Acta Anaesthesiol Scand 56: 465-473, 2012.

23. Mi WD, Sakai T, Takahashi S and Matsuki A: Haemodynamic and electroencephalograph responses to intubation during induction with propofol or propofol/fentanyl. Can J Anaesth 45: 19-22, 1998.

24. Guignard B, Menigaux C, Dupont X, Fletcher D and Chauvin M: The effect of remifentanil on the bispectral index change and hemodynamic responses after orotracheal intubation. Anesth Analg 90: 161-167, 2000.

25. Ekman A, Lindholm ML, Lennmarken C and Sandin R: Reduction in the incidence of awareness using BIS monitoring. Acta Anaesthesiol Scand 48: 20-26, 2004.

26. Sugiura S, Hidaka K, Seki S and Tsuchida H: Comparative study of two beta adrenergic antagonists on hemodynamics at the endotracheal intubation. Anesthesiology 107: A309, 2007.

27. Messieha ZS, Guirguis S and Hanna S: Bispectral index monitoring (BIS) as a guide for intubation without neuromuscular blockade in office-based pediatric general anesthesia: A retrospective evaluation. Anesth Prog 58: 3-7, 2011.

28. Messner M, Beese U, Romstöck J, Dinkel M and Tschaikowsky K: The bispectral index declines during neuromuscular block in fully awake persons. Anesth Analg 97: 488-491, 2003.

29. Albertin A, Casati A, Federica L, Roberto V, Travaglini V, Bergonzi P and Torri G: The effect-site concentration of remifentanil blunting cardiovascular responses to tracheal intubation and skin incision during bispectral index-guided propofol anesthesia. Anesth Analg 101: 125-130, 2005. 